\title{
Numerical Simulation of Pedestrian Flow Based on A Higher-Order Macroscopic Model
}

\author{
Y.Q. Jiang \\ Department of Mathematics
}

Southwest University of Science and Technology

Mianyang, China

\begin{abstract}
In this paper, a higher-order macroscopic model is applied to simulate non-equilibrium pedestrian flow. The path choice behavior is described by a time dependent Hamilton Jacobi equation based on the assumption that pedestrian flow always tends to walk along a path with lowest actual walking cost. A self-adaptive method of successive averages coupled with a cell-centered finite volume method is used to solve the macroscopic model. A numerical example is designed to investigate macroscopic features and route choice behavior of pedestrian flow walking in a channel scattered with an obstruction.
\end{abstract}

Keywords-higher-order model, non-equilibrium pedestrian flow; path choice behaviour; method of successive averages; macroscopic features

\section{INTRODUCTION}

In recent decades, pedestrian traffic has attracted attention of numerous scholars [1-5]. Many typical dynamical phenomena in pedestrian dynamics, such as clustering at bottlenecks, stripe formation and chevron effect, etc [1], can be perfectly captured by physical methods. These studies can help to develop guidelines for planning and designing pedestrian facilities for public transport planners as well as infrastructure designers.

Many microscopic models, such as the social force model [2], cellular automata model [3], the lattice gas model [4], have been presented to investigate the collective phenomena in pedestrian traffic. Macroscopic models treat a large group of pedestrians as a flowing continuum and are generally described as a system of partial differential equations (PDEs) [5]. These models are useful for measuring macroscopic variables of pedestrian density, speed, and flow. For equilibrium traffic flow, there are two traffic assignment patterns, namely user equilibrium (UE) and system optimum (SO) [6-8]. The UE models encompass predictive UE models [9], in which pedestrians have predictive information when they are making a path-choice decision, and reactive dynamic user equilibrium models $[7,10]$, in which pedestrians have to rely on the instantaneous information available to them and make their choices.

In this paper, a higher-order macroscopic model is developed to illustrate non-equilibrium pedestrian flow. It is assumed that pedestrian flow always tends to choose a path

\author{
S.G. Zhou
}

China Aerodynamics Research and Development Center

Mianyang, China

from the current position and time to the destination with lowest actual walking cost, which is corresponding to the predictive UE pattern. This path choice behavior is described by a time dependent Hamilton Jacobi (HJ) equation. The model is solved by a cell-centered finite volume (FV) method and a self-adaptive method of successive averages (MSA). A numerical experiment is carried out to investigate macroscopic features and route choice behavior of pedestrian flow walking in a channel with an obstacle.

\section{II.MATHEMATICAL MODEL}

Consider a large group of pedestrians walking in a $2 \mathrm{D}$ continuous domain $\Omega$. The boundary of $\Omega$ consists of inflow boundary $\Gamma_{i}$, outflow boundary $\Gamma_{\circ}$, and solid wall boundary ${ }^{{ }}$. The density and average velocity of pedestrian flow are represented by $\rho(x, y, t)$ (in ped $/ \mathrm{m} 2$ ) and $\mathbf{v}(x, y, t)=(u(x, y, t), v(x, y, t))$ (in $\mathrm{m} / \mathrm{s})$, respectively. Let $\mathbf{e}(x, y, t)=\left(e_{1}(x, y, t), e_{2}(x, y, t)\right)$ be the desired direction of motion of pedestrian flow. $T=\left[0, t_{\text {end }}\right]$ (in s) is the modeling period.

Based on continuum dynamics, the governing equations of non-equilibrium pedestrian flow in $\Omega$ can be described as Euler equations with relaxation [11]:

$$
\left\{\begin{array}{c}
\frac{\partial \rho}{a}+\nabla \cdot \rho \mathbf{v}=0, \\
\frac{\hat{\alpha}}{\hat{a}}+(\mathbf{v} \cdot \nabla) \mathbf{v}+c^{2}(\rho) \frac{\nabla \rho}{\rho}=\frac{U(\rho) \mathbf{l}-\mathbf{v}}{\tau},
\end{array}\right.
$$

which is subject to the initial conditions:

$$
\rho(x, y, 0)=\rho_{0}(x, y), \mathbf{v}(x, y, 0)=\mathbf{v}_{0}(x, y) .
$$

In Eqs. (1), $\tau=0.5 s$ is the characteristic relaxation time, $c(\rho)$ is the propagating speed of small perturbation in pedestrian flow which is equivalent traffic sonic speed, and $c^{2}(\rho) \frac{\nabla \rho}{\rho}$ is called the anticipation term which reflects pedestrian reaction to the surrounding pedestrians. $U_{e}(\rho)$ 
describes an equilibrium state of the speed-density $\underline{U_{e}(\rho) \mathbf{e}-\mathbf{v}}$

relationship and $\tau$ is called the relaxation term which represents a relaxation to the equilibrium. By different definitions of $c(\rho), U_{e}(\rho)$, and $\mathbf{e}(x, y, t)$, we can obtain different macroscopic dynamic models for nonequilibrium pedestrian flow.

We define traffic pressure $P(\rho)$ as a function of the pedestrian density, which describes the response of pedestrians to compression and is expressed by

$$
\frac{d P(\rho)}{d \rho}=c^{2}(\rho)
$$

The conservation form of Eqs. (1) is written as

$$
\frac{\partial Q}{\partial t}+\nabla \cdot(F, G)=
$$

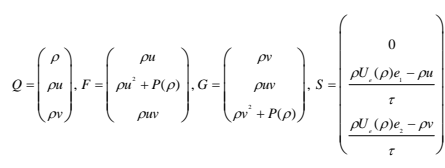

The eigenvalues

of Jacobian matrices $A(Q)=\frac{\partial F}{\partial Q}, B(Q)=\frac{\partial G}{\partial Q}$ are

$$
\begin{aligned}
& \lambda_{1}^{A}=u-c(\rho), \lambda_{2}^{A}=u, \lambda_{3}^{A}=u+c(\rho), \\
& \lambda_{1}^{B}=v-c(\rho), \lambda_{2}^{B}=v, \lambda_{3}^{B}=v+c(\rho) .
\end{aligned}
$$

Let $C(x, y, t)$ be the local travel cost distribution and is defined by

$$
C(x, y, t)=\frac{1}{U_{e}(\rho(x, y, t)}
$$

It is presumed that pedestrians have predictive information about traffic conditions over time and the desired direction of pedestrian movement is always to minimize the actual travel cost from the current position and time to the destination, thus resulting in a predictive UE pattern for the dynamic system [6,9].

Let $\Phi(x, y, t)$ (in s) be cost potential which describes the lowest cost incurred by a pedestrian walking from the origin $(x, y) \in \Omega$ at time $\mathrm{t}$ to the destination $\Gamma_{0}$ at which $\Phi(x, y, t)=0$. For the predictive UE pattern, the optimum motion trajectory can be uniquely determined as [9]:

$$
\mathbf{e}=-\frac{\nabla \Phi}{\|\nabla \Phi\|}
$$

Here, $\Phi$ satisfies a time dependent HJ equation:

$$
\frac{\partial \Phi}{\partial t}=U_{e}(\|\nabla \Phi\|-C)
$$

Assuming that all pedestrians have left the modeling domain and that there is no traffic at $t=t_{\text {end }}$, the traffic state is considered to be static and the travel cost to the destination is the instantaneous cost, i.e. $\Phi\left(x, y, t_{\text {end }}\right)=\Phi_{0}(x, y)$. Here, $\Phi_{0}(x, y)$ satisfies an Eikonal equation:

$$
\left\{\begin{array}{c}
\left\|\Phi_{0}(x, y)\right\|=C\left(x, y, t_{\text {end }}\right) \\
\Phi(x, y, t)=0, \forall(x, y) \in \Gamma
\end{array}\right.
$$

\section{NUMERICAL METHOD}

From Section 2, the higher-order macroscopic model of non-equilibrium pedestrian flow can be written in two parts. The one part is conservation laws (4) which satisfy the initial conditions (2). The another part is the time dependent HJ equation (7) which is subject to the initial and boundary conditions $\Phi\left(x, y, t_{\text {end }}\right)=\Phi_{0}(x, y), \forall(x, y) \in \Omega \quad$ and $\Phi(x, y, t)=0, \forall(x, y) \in \Gamma_{。}$. The difficulty about the numerical computation of the model is the initial condition of the HJ equation (7) is obtained at $t=t_{\text {end }}$, as opposed to that of Eqs. (4) at $t=0$. To solve this problem, we use a first order cell-centered FV scheme to solve Eqs. (4) and (7), the fast sweeping method to solve Eq. (8), and the self-adaptive MSA [9] to solve the discrete fixed-point problem.

The treatment of source terms in Eqs. (4) involves a fractional step method, by which each time step $\Delta t$ is split into three steps. Eqs. (4) are split into the ordinary differential equations (ODEs) and the PDEs, i.e.

$$
\frac{d Q}{d t}=S, \quad \frac{\partial Q}{\partial t}+\nabla \cdot(F, G)=0 .
$$

The solution values of $Q^{n+1}$ at the n+1-th time step are obtained from $Q^{n}$ at the n-th time step as follows. Firstly, we update $Q^{n}$ with $\tilde{Q}^{n}$ by applying a classical implicit Euler method to the ODEs with the time increment $\Delta t / 2$. Secondly, we update $\tilde{Q}^{n}$ with $\bar{Q}^{n}$ by applying the LaxFriedrichs scheme to the PDEs with the time increment $\Delta t$. 
Finally, we update $\bar{Q}^{n}$ with $Q^{n+1}$ by applying the implicit Euler method to the ODEs with the time increment $\Delta t / 2$.

The first order cell-centered FV scheme for Eqs. (4) is written as

$$
Q_{i j}^{n+1}=Q_{i j}^{n}-\frac{\Delta t}{\Delta x}\left(\hat{F}_{i+\frac{1}{2},}-\hat{F}_{i-\frac{1}{2}}\right)-\frac{\Delta t}{\Delta y}\left(\hat{G}_{i+\frac{1}{2}}-\hat{G}_{i-\frac{1}{2}}\right)
$$

where $^{\Delta x, \Delta y}$ are the mesh sizes in the $\mathrm{x}$ - and $\mathrm{y}$-direction, $\hat{F}_{i+\frac{1}{-j}}, \hat{Q}_{i j+\frac{1}{2}}$

respectively. $\quad{ }_{2}^{i+-j}{ }_{2}^{i j+-}$ are the Lax-Friedrichs numerical fluxes in the $\mathrm{x}$ - and $\mathrm{y}$-direction, respectively, and are expressed by

$$
\begin{aligned}
& \hat{F}_{i+1, j}=\frac{1}{2}\left(F\left(Q_{i j}^{n}\right)+F\left(Q_{i t 1 j}^{n}\right)-\alpha_{x}\left(Q_{i t i j}^{n}-Q_{i j}^{n}\right)\right), \hat{Q}_{j+1}=\frac{1}{2}\left(G\left(Q_{i j}^{n}\right)+G\left(Q_{i j+1}^{n}\right)-\alpha_{y}\left(G_{i t+1}^{n}-G_{i j}^{n}\right)\right), \\
& \alpha_{x}=\max (|u|+C) \text { and } \alpha_{y}=\max (|v|+C)
\end{aligned}
$$$$
\text { ere }
$$

We define $H=U_{e}(\|\nabla \Phi\|-C)$ and thus the first order cell-centered FV scheme for Eq. (7) is given by

$$
\Phi_{i j}^{n}=\Phi_{i j}^{n+1}-\Delta t \hat{H}\left[\left(\Phi_{x}\right)_{i j}^{-},\left(\Phi_{x}\right)_{i j}^{+},\left(\Phi_{y}\right)_{i j}^{-},\left(\Phi_{y}\right)_{i j}^{+}\right]
$$

where

$\left(\Phi_{x}\right)_{i j}^{-}=\frac{\Phi_{i j}^{n+1}-\Phi_{i-1 j}^{n+1}}{\Delta x},\left(\Phi_{x}\right)_{i j}^{+}=\frac{\Phi_{i+1 j}^{n+1}-\Phi_{i j}^{n+1}}{\Delta x},\left(\Phi_{y}\right)_{i j}^{-}=\frac{\Phi_{i j}^{n+1}-\Phi_{i j-1}^{n+1}}{\Delta y},\left(\Phi_{y}\right)_{i j}^{+}=\frac{\Phi_{i j+1}^{n+1}-\Phi_{i j}^{n+1}}{\Delta y}$ $\hat{H}\left(u^{+}, u^{-}, v^{+}, v^{-}\right)$is the Lax-Friedrichs numerical flux and is defined by

$$
\hat{H}\left(u^{+}, u^{-}, v^{+}, v^{-}\right)=\hat{H}\left(\frac{u^{+}+u^{+}}{2}, \frac{v^{+}+v^{-}}{2}\right)-\frac{1}{2} \alpha_{x}^{u}\left(u^{+}-u^{-}\right)-\frac{1}{2} \alpha_{y}^{u}\left(v^{+}-v^{-}\right)
$$

where

$$
\alpha_{x}^{H}=\max _{A \leq u \leq B} H_{1}(u, v), \alpha_{y}^{H}=\max _{A \leq u \leq B} H_{2}(u, v)
$$

$H_{1}=\frac{\partial H}{\partial \Phi_{x}}, H_{2}=\frac{\partial H}{\partial \Phi_{y}},[\mathrm{~A}, \mathrm{~B}]$ is the value range of $\boldsymbol{U}^{ \pm}$and
Here, $[\mathrm{C}, \mathrm{D}]$ is the value range of $v^{ \pm}$.

Note that Eqs. (9) and (10) cannot be solved together as they have different initial times. Therefore, the discrete model is a fixed-point problem that can be solved by the MSA. See [9] for the description of MSA in detail.

\section{NUMERICAL EXPERIMENTS AND RESULTS}

We consider a large group of pedestrians walking in a channel of size $100 \mathrm{~m} \times 50 \mathrm{~m}$ scattered with a square obstruction of size $20 \mathrm{~m} \times 20 \mathrm{~m}$ centered at $(50 \mathrm{~m}, 20 \mathrm{~m})$. The entrance and exit with width of $50 \mathrm{~m}$ and $30 \mathrm{~m}$ are set at the left boundary $\Gamma_{i}$ and the right boundary $\Gamma_{o}$ of the channel, respectively. The study horizon $\mathrm{T}$ is set to $240 \mathrm{~s}$. The speeddensity relationship $U_{e}(\rho)$ is given by

$$
U_{e}(\rho)=v_{1} \exp \left(-\gamma \rho^{2}\right),
$$

with the free flow speed $v_{f}=1.034 \mathrm{~m} / \mathrm{s}$ and the model parameter $\gamma=0.075$. The critical density dividing the uncongested and congested density regions is $\rho_{c}=\rho_{\max } / \sqrt{2 \gamma} \approx 2.58 \mathrm{ped} / \mathrm{m}^{2}$. The traffic pressure $P(\rho)$ is defined as $P(\rho)=c_{0} \rho^{2}$ with an anticipation coefficient $c_{0} \geq 0$.

The initial and boundary conditions for the macroscopic model are described as follows. Initially, $\rho_{0}=0 \mathrm{ped} / \mathrm{m} 2$ and $\mathbf{v}_{0}=\mathbf{0} \mathrm{m} / \mathrm{s}$. At entrance $\Gamma_{i}$, a free and equilibrium inflow is given by

$$
\rho=\left\{\begin{array}{cl}
\rho_{m} \frac{t}{60} & , t \in[0 s, 60 s] \\
\rho_{m} \frac{(t-120)}{60} & , t \in[60 \mathrm{~s}, 120 \mathrm{~s}], \rho_{m}=2.0 \mathrm{ped} / \mathrm{m}^{2},(u, v)=\left(U_{e}(\rho), 0\right) \\
0 & , t \in[120 \mathrm{~s}, 240 \mathrm{~s}]
\end{array}\right.
$$

At the solid walls $\Gamma_{w}$, the free-slip and non-permeable boundary conditions are applied with $\frac{\partial \rho}{\partial \mathbf{n}}=0, \mathbf{v} \square \mathbf{n}=0$. the outflow boundary $\Gamma_{o}, \frac{\partial \rho}{\partial \mathbf{n}}=0, \mathbf{v}\left\lceil\mathbf{n}=v_{f}\right.$.

Figure. 1 plots the density distribution of pedestrian flow walking in the domain scattered with an obstruction in the four phases with the parameter $\mathrm{c} 0=2.0$. In the first phase, pedestrian flow is divided into two sub-streams by the obstruction and a triangular vacuum region is formed on the left-hand side of the obstruction, which is consistent with the route choice strategy of pedestrians. In the second phase, the triangular vacuum region has narrowed dramatically with the increase of pedestrians near the obstruction. In the third phase, the density of pedestrian flow increases steadily around the obstruction and almost reaches a peak value. Two high density regions (i.e. two shocks) which represent traffic congestion are seen near the two left-hand corners of the obstruction because of the shortened width of the channel. The previous triangular vacuum region has disappeared and another triangular vacuum region is formed on the right-hand side of the obstruction. In the fourth phase, pedestrian flow has passed the obstruction and meanwhile gathered near the exit.

Figure. 2 depicts the density distributions for the dissipation of pedestrian flow in four phases with the parameter $\mathrm{c} 0=0.25$. The first and second phases (see Figure. 2 (a) and (b)) demonstrate almost the same results as in the corresponding sub-figures of Fig. 1 . In the third phase, the areas of the high density regions are much bigger than those 


\begin{tabular}{|c|c|c|c|c|}
\hline $\mathrm{t}$ & $30 \mathrm{~s}$ & $60 \mathrm{~s}$ & $120 \mathrm{~s}$ & $180 \mathrm{~s}$ \\
\hline $\mathrm{c}_{0}=4.0$ & 0.916 & 1.957 & 2.881 & 2.345 \\
\hline $\mathrm{c}_{0}=1.0$ & 0.973 & 2.372 & 4.087 & 2.845 \\
\hline $\mathrm{c}_{0}=0.125$ & 0.983 & 2.732 & 5.328 & 3.724 \\
\hline
\end{tabular}

observed in Figure. 1 (c), which indicates more serious traffic congestion appears. The production mechanism of more serious traffic jam is that the lower sonic speed at which the large disturbance caused by the obstruction spreads across the domain. The two shocks with the lower sonic speed are stronger than those with the faster one. In the fourth phase, high density regions are also observed near the two corners of the exit.

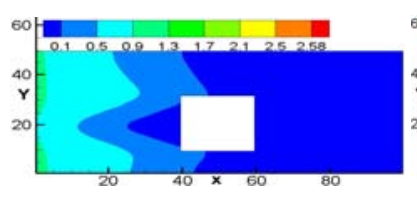

(a) $30 \mathrm{~s}$

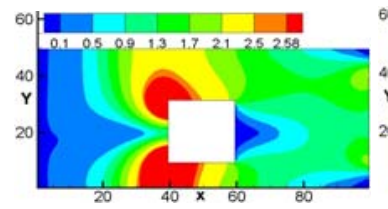

(c) $120 \mathrm{~s}$

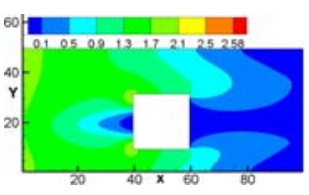

(b) $60 \mathrm{~s}$

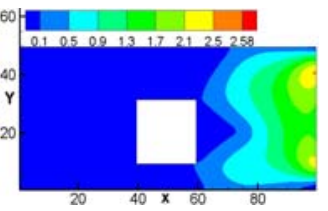

(d) $180 \mathrm{~s}$
FIGURE I. FIGURE I: DENSITY PLOT AT DIFFERENT TIMES WITH $\mathrm{C}_{0}=2$.

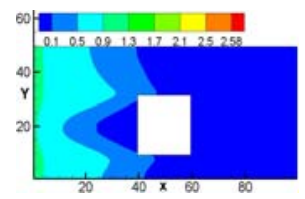

(a) $30 \mathrm{~s}$

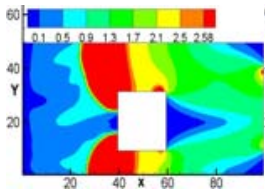

(c) $120 \mathrm{~s}$

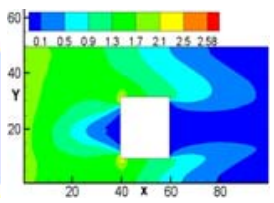

(b) $60 \mathrm{~s}$

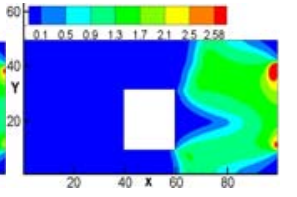

(d) $180 \mathrm{~s}$
FIGURE II. DENSITY PLOT AT DIFFERENT TIMES WITH C0=0.25.

Table 1 shows the peak values of the density at different times, with $\mathrm{c} 0=4.0,1.0$, and 0.125 , respectively. It is observed that pedestrians are discharged more smoothly with the increase of the anticipation coefficient $\mathrm{c} 0$. Fig. 3 shows the densities of pedestrian flow along $x=37 \mathrm{~m}$ and $y=33 m$, which further illustrates the densities become smoother with large $\mathrm{c} 0$. Furthermore, the strong response of pedestrians to compression can prevent traffic congestion and collision accidents from occurring effectively.

\section{TABLEI. The MAXimum Densities With DifFERENT VALUES OF $\mathrm{C}_{0}$.}

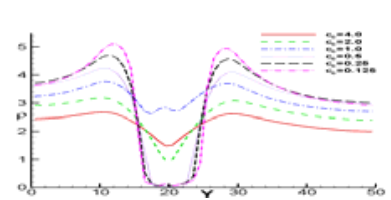

(a) $\mathrm{x}=37 \mathrm{~m}$

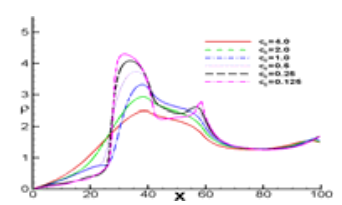

(b) $y=33 m$
FIGURE III. DENSITIES ALONG X = 37M AND Y = 33M WITH DIFFERENT VALUES OF C0 AT TIME T=120S.

\section{SUMMARY}

In this work, a higher-order pedestrian flow model composed of Euler equations with relaxation and a time dependent HJ equation is presented. Numerical results show that this model can reproduce some macroscopic features of pedestrian flow, such as the spatio-temporal distribution of density. Large anticipation coefficient or high traffic pressure could prevent from traffic congestion.

\section{ACKNOWLEDGEMENTS}

This work was supported by the Research Foundation of SWUST (No. 10zx7137) and NSFC grants (No. 11202175 and No. 11372294).

\section{REFERENCES}

[1] J. Cividini, H.J. Hilhorst, C. Appert-Rolland: Journal of Physics A Vol. 46 (2013) , p. 345002

[2] D. Helbing, I. Farkas, T. Vicsek: Nature Vol. 407 (2000), p. 487

[3] A. Kirchner, A. Schadschneider: Journal of Physics A Vol. 312(2002), p. 260

[4] W. Song, X. Xu, B.H. Wang, S. Ni. Journal of Physics A, 492 (2006), p. 363

[5] R.L. Hughes: Transportation Research Part B 36 (2002), p. 507

[6] Y.Q. Jiang, S.C. Wong, H.W. Ho, P. Zhang, R.X. Liu and A. Sumalee: Transportation Research Part B Vol. 45(2011), p. 343

[7] Y.Q. Jiang, T. Xiong, S.C. Wong, C.W. Shu, M.P. Zhang, P. Zhang, W.H.K. Lam: Acta Mathematica Sinica Vol. 29B(2009), p. 1541

[8] Y.Z. Tao, Y.Q. Jiang, J. Du, S.C. Wong, P. Zhang, Y.H. Xia and K. Choi: Journal of Advanced Transportation (2013), in press.

[9] J. Du, S.C. Wong, C.W. Shu, T. Xiong, M.P. Zhang, K. Choi: Transportation Research Part B Vol. 56(2013), p. 96

[10] L. Huang, S.C. Wong, M.P. Zhang, C.W. Shu and W.H.K. Lam: Transportation Research Part B Vol. 43(2009), p. 127

[11] Y.Q. Jiang, P. Zhang, S.C. Wong and R.X. Liu: Journal of Physics A 389(2010), p. 4623 